\title{
Improved system for identifying biological tissue temperature using electrical impedance tomography
}

\author{
Evgeniy Korolyuk*, and Konstantin Brazovskii \\ National Research Tomsk Polytechnic University, 634050 Tomsk, Russia
}

\begin{abstract}
This paper proposes a cheap and compact medical system that determines the temperature of an object using broadband impedance tomography. This system can be used in medicine to visualize ice structure in tissue during cryosurgical operations, as well as for fault diagnosis and location in studied industrial objects. These effects are achieved by measuring electrical impedance between electrode pairs in the measuring chamber. The assembled prototype is compact, consumes little power, and allows to non-invasively determine the impedance of a target object in real time. The research included experimental studies to determine the dependence of the impedance spectrum of saline water and muscle tissue on temperature in broad band spectrum, which allowed to obtain the dependence of total electrical impedance of target objects on temperature.
\end{abstract}

\section{Introduction}

One of the main problems in the medical use of freezing is the difficulty of determining the boundaries and depth of cryotherapy. Due to individual characteristics of tissue, different cooling rates, and high temperature gradients during freezing, it is not always possible to accurately determine freezing depth [1].

However, regardless of the apparent simplicity of the method, the procedure of cryodestruction does not guarantee the complete removal of abnormal tissue. Insufficient influence intensity leads to local and systemic complications, which is a direct indication for reoperation. The main cause of complications is incomplete removal of abnormal biological tissues due to insufficient intensity of cryotherapy. The complexity of correct choice of cryotherapy mode is conditioned by the fact that methods of assessing depth and time of freezing are insufficiently accurate and largely subjective. The first reason of high error probability lies in the broad range of difference in water content and electrolyte concentration in tissues of different species, which leads to significant differences in physical properties (heat capacity, thermal conductivity, and freezing temperature). The second reason is conditioned by a significant temperature gradient between the surface and internal areas of biological objects [2].

*Corresponding author: esk13@tpu.ru 
It is obvious that increasing the accuracy of determining freezing depth requires more advanced methods. One of the most promising methods of determining the degree of tissue damage and cryonecrosis is measuring the active component of tissue impedance. Freezing of aqueous solutions in cells leads to a sharp increase of electrical impedance, which is a reliable indicator of the quality of cryodestruction process [3].

This paper describes the work carried out to design a low-cost medical device that uses electrical impedance tomography to determine the temperature of a target object and allows to visualize the formation of ice structures within the tissue.

\section{Related work}

At the moment, there exist several visualizing methods of diagnostics that allow to construct images of internal environments of a biological object. The main types of such imaging are the methods of magnetic resonance imaging (MRI) and computed tomography (CT).

\subsection{Magnetic resonance and computed tomography.}

The magnetic resonance tomography method is a method of obtaining images of biological objects using the phenomenon of nuclear magnetic resonance. Modern magnetic resonance scanners allow to non-invasively study internal organs, as well as various processes inside them.

The computed tomography method is a method of obtaining images of biological objects by determining the difference of attenuation of X-ray radiation depending on tissue density. Just like magnetic resonance imaging, this method allows to study internal organs and various processes inside them.

However, every method has its limitations and drawbacks. The intense vortex magnetic field during magnetic resonance scanning imposes extreme requirements to the scanner and other equipment in the scanning room, as well as to the room itself. The principle of computed X-ray tomography is based on using X-ray radiation. This results in the possibility of a patient contracting cancerous diseases.

It should be noted that given the ample capabilities of MRI and CT (both of these methods provide the most comprehensive assessment of a patient's organism condition), these procedures are still more expensive compared to conventional X-ray or ultrasonography. High costs of diagnostics, the necessity to carry it out in real time, and use of metal tips in virtually all contemporary cryotherapy tools make the use of MRI and CT impractical for high-speed cryotherapy.

In order to eliminate the limitations described above, this study employs another less costly method that does not require a special room and maintenance staff. Most of the drawbacks and limitations described can be using another method of tomographic imaging: electrical impedance tomography.

\subsection{Electrical impedance tomography}

Electrical impedance (or simply impedance) tomography is the name for a technique of obtaining body section images by means of electrical sounding, calculations and algorithms of reconstruction of impedance distribution (resistance of different organs to electric current).

This scanning method stood out in the late 80 ies - early 90 ies of the past century. The essence of the method lies in the phenomenon that when alternating electric current passes 
through a biological object, evoked potentials are registered on its surface. Spatial distribution of electrical conductivity inside the target object can be identified if the value of the applied current and the potential registered on the object's surface are known.

\section{Implementation}

\subsection{Electrodes and measuring chamber}

In order to carry out experiments, a measuring chamber was constructed that houses a target object. Recording electrodes are located on the internal faces of the measuring chamber as shown in Figure 1 (right). The electrodes are made of silver foil coated with $\mathrm{AgCl}$ (silver chloride). The choice of silver-chloride electrodes is conditioned by their low level of polarization and baseline drift, low noise level, and impedance to frequency stability.

\subsection{Control board}

The main element of the control board designed during this study (Figure 1, left) is an STM32f1 microcontroller built on a Cortex M3 core and working with the frequency of 72 MHz. The DMA mode was used to ensure the high speed of digital-to-analog converter (DAC) operation. A 14-bit AD9764 DAC by Analog Devices was chosen as the source of scanning current [4]. Due to high output impedance of the DAC (around $100 \mathrm{kOhm}$ ) and to ensure the maximum voltage linearity between the DAC output and target object, it is necessary to use a current repeater with broad operating band of output voltage and higher output impedance. An E20-10 high-speed analog-to-digital converter (ADC) module was used to measure the electric potential difference between the electrodes in the measuring chamber. This module is a four-channel, 14-bit ADC with the maximum conversion frequency of $10 \mathrm{MHz}$.
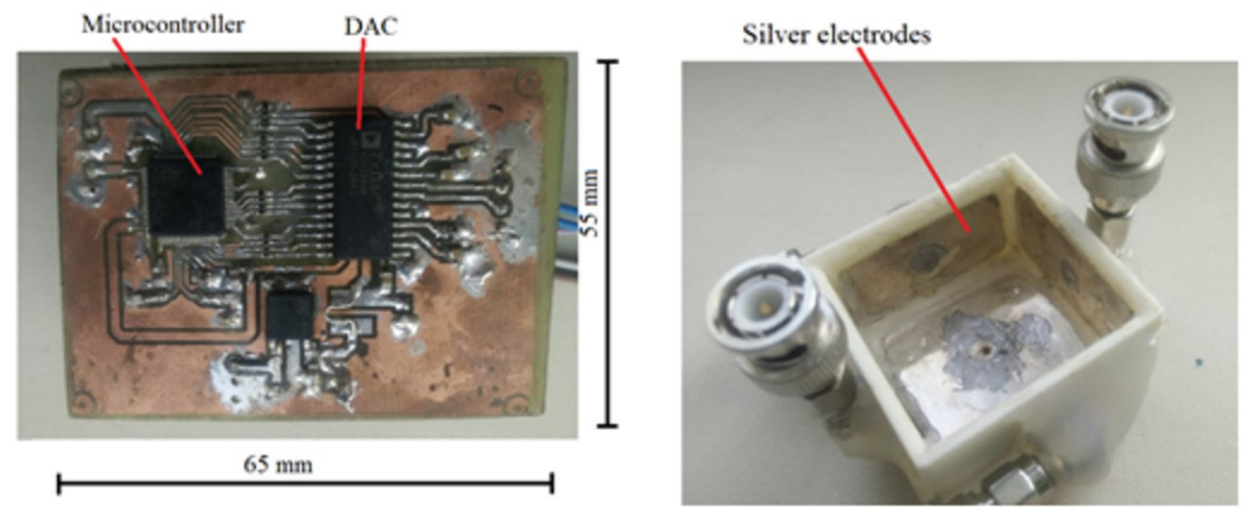

Fig. 1. Control board (left) and measuring chamber (right).

\section{Results and discussion}

In order to check the assembled device, it was tested using resistors with the resistance range of 50 to $500 \mathrm{Ohm}$. It is obvious that with the increase in resistance, the voltage in a resistor further drops and, consequently, power spectral density increases. The obtained results were united into a single chart as shown in Figure 3. 


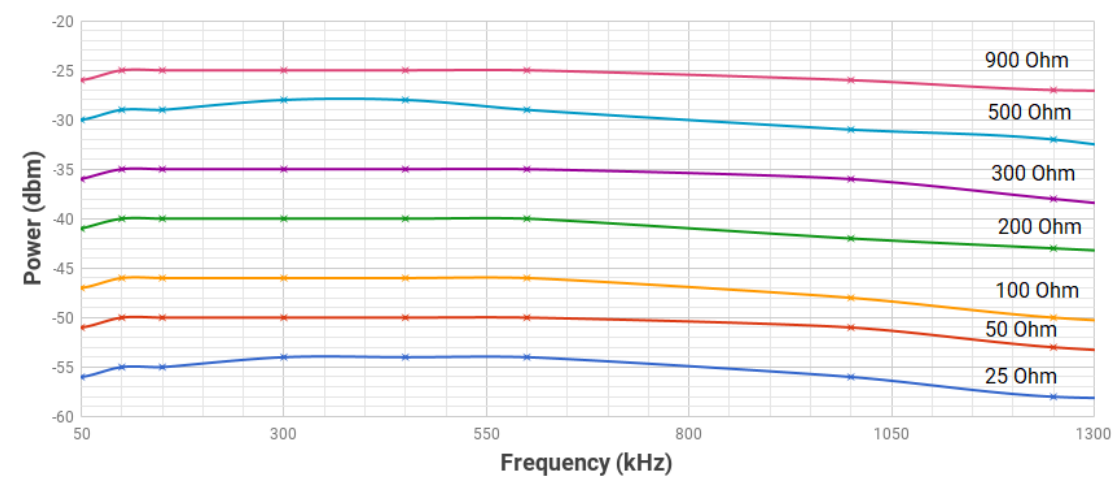

Fig. 2. Power spectral density of fixed-value resistors. The current strength is $1 \mathrm{~mA}$ (amplitude value). The $\mathrm{Y}$ axis is the power in milliwatts in the logarithmic scale of the measurement; the $\mathrm{X}$ axis is the frequency in $\mathrm{kHz}$.

\subsection{Bioimpedance measurement}

Further experiments were dedicated to studying the dependence of bioimpedance on temperature in a broad band range (from $50 \mathrm{kHz}$ to $2500 \mathrm{kHz}$ ). Target samples were represented by water with dissolved salts and muscle tissue. Temperature in the target samples varied in different range from 20 below zero to 25 above zero degrees Celsius as shown in Figures 3 and 4.

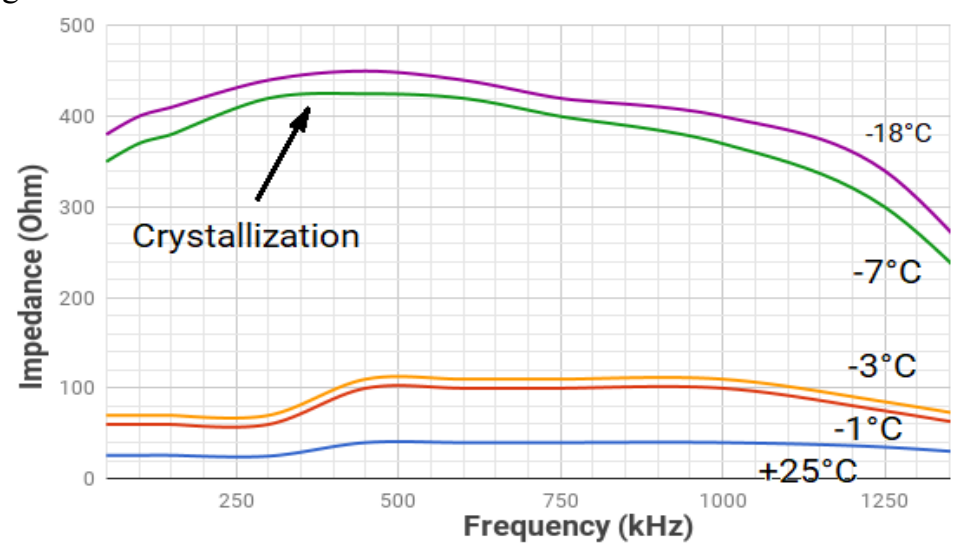

Fig. 3. Bioimpedance spectrum of water with dissolved salts with temperature change. The current strength is $1 \mathrm{~mA}$ (amplitude value). The $\mathrm{Y}$ axis is the impedance in $\mathrm{Ohm}$; the $\mathrm{X}$ axis is the frequency in $\mathrm{kHz}$. 


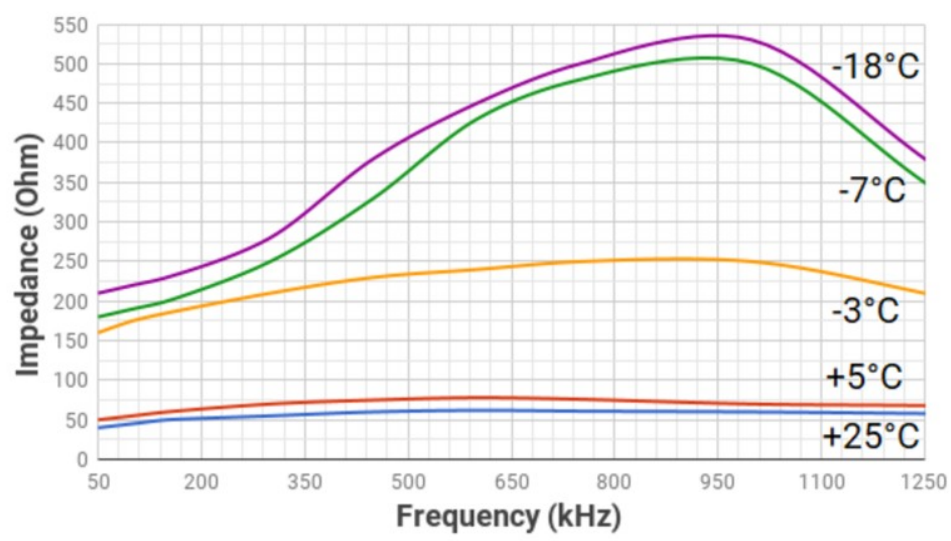

Fig. 4. Bioimpedance spectrum of muscle tissue with temperature change. Current strength: $1 \mathrm{~mA}$ (amplitude value). The $\mathrm{Y}$ axis is the impedance in $\mathrm{Ohm}$; the $\mathrm{X}$ axis is the frequency in $\mathrm{kHz}$.

\section{Conclusion}

This study presented a laboratory prototype of a device that uses electrical impedance tomography to identify temperature and impedance of a target object. Measurements are carried out using five electrodes located in the measuring chamber. After performing 30 measurements between two pairs of electrodes, it became possible to estimate the impedance of the target object. The experimental studies resulted in obtaining dependencies of the bioimpedance spectrum on the temperature of aqueous solutions of mineral salts and muscle tissue in the temperature range of -18 to +25 degrees Celsius. Using reference values of resistance, the accuracy of the prototype could be tested. The obtained results show that decrease of temperature leads to increase in impedance in the frequency range of 50 to $1300 \mathrm{kHz}$ for aqueous solution of mineral salts and for muscle tissue. Peak values are located in the frequency of $375 \mathrm{kHz}$ for aqueous solution and $950 \mathrm{kHz}$ for muscle tissue. The data collected allow to reliably identify the degree of biological material freezing by measuring electrical impedance in the frequency band from 50 to $1300 \mathrm{kHz}$.

\section{References}

1. P. Laugnier, G. Berger. Assessment of echography as a monitoring technique for cryosurgery. Ultrason Imaging 15(1), 14 (1993)

2. J. F. Edd, L. Horwitz, and B. Rubinsky, Temperature dependence of tissue impedivity in electrical impedance tomography of cryosurgery. IEEE Trans. Biomed. Eng. 52(4), 695 (2005)

3. T. K. Bera , Bioelectrical Impedance Methods for Noninvasive Health Monitoring: A Review. Journal of Medical Engineering 2014, 381251, 28 (2014)

4. Analog Devices Inc. http://www.analog.com/media/en/technical-documentation/datasheets/AD9764.pdf 